\title{
Caracterización microscópica de texturas superficiales fabricadas aditivamente mediante estereolitografía láser
} stereolithography

\author{
Caracterização microscópica de texturas superficiais fabricadas aditiva por \\ estereolitografia laser
}

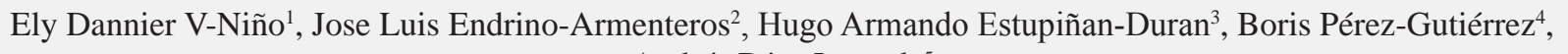
Andrés Díaz-Lantada ${ }^{5}$

Forma de citar: E. D. V-Niño, J. L. Endrino-Armenteros, H. A. Estupiñan-Duran, B. Pérez-Gutiérrez, A. DíazLantada, "Caracterización microscópica de texturas superficiales fabricadas aditivamente mediante estereolitografía láser”, Respuestas, vol. 21, no. 2, pp. 37-47, 2016.

Recibido:

Febrero 17 de 2016

Aceptado:

Junio 6 de 2016

${ }^{1}$ Magister en Física. deydannv@gmail.com Orcid: 0000-0003-4154-7179

Universidad Politécnica de Madrid

Madrid, España Universidad Industrial de Santander

Foundation of Researchers in Science and Technology of Materials

Bucaramanga-Colombia

${ }^{2}$ Doctorado en Ingeniería Mecánica

j.1.endrino@cranfield.ac.uk Orcid: 0000-0002-3084-7910 Cranfield University Bedfordshire-United Kingdom.

${ }^{3}$ Doctorado en Ingeniería Química

haestupinand@unal.edu.co Orcid: 0000-0002-9607-3364

Universidad Nacional de Colombia

Medellín-Colombia.

${ }^{4}$ Magister en Ingeniería de Sistemas

borisperezg@ufps.edu.co Orcid: 0000-0001-9249-1756 Universidad Francisco de Paula Santander

San José de Cúcuta-Colombia.

${ }^{5}$ Doctorado Ingeniero Industrial adiaz@etsii.upm.es Orcid: 0000-0003-3947-6113

Universidad Politécnica de Madrid

Madrid-España.

\section{Resumen}

Objetivo: En nuestro trabajo presentamos el desarrollo de texturas superficiales con diferentes geometrías fabricadas por manufactura aditiva. Metodología: Los sustratos con diferentes texturas superficiales son diseñados por medio de programas asistidos por computador (CAD). La fabricación de las diferentes superficies se realiza capa a capa, en un solo proceso, por medio de la técnica de estereolitografía láser (SLA), directamente desde los archivos CAD. Resultados: Las superficies de los sustratos fueron evaluadas mediante ensayos ópticos con el objetivo de medir la topografía de las superficies, validar el acabado superficial y controlar los métodos de fabricación a través de las estrategias de medición en diferentes perfiles. Conclusión: En este estudio mostramos que las texturas superficiales impresas presentaron una reducción de los valores de medidas de longitud, volumen y masa en comparación con la definida en el diseño.

Palabras clave: Manufactura aditiva, Microscopía óptica, Resina fotocurable.

\begin{abstract}
Objective: This work shows surface texture development using several different geometries produced by additive manufacturing. Methodology: The substrates with different surface textures are designed by computer aided programs (CAD). Manufacture of the different surfaces is performed in a layer by layer basis, in a single process using the laser stereolithography technique (SLA), directly from CAD files. Results: substrates surfaces were evaluated by optical tests in order to measure the topography of such surfaces, to validate the surface finishing and to control manufacturing methods by using the strategy of measurement in different profiles. Conclusion: In this study we demonstrated that the printed surface textures showed a reduction in the values of length, volume and mass measurements when compared to the ones defined in the design.
\end{abstract}

Keywords: Additive Manufacturing, optical microscopy, photo-curable resin.

\section{Resumo}

Objetivo: Em nosso trabalho, apresentamos o desenvolvimento de texturas de superfície com diferentes geometrias produzidas por manufatura aditiva. Metodologia: Os 
No. 2

Jul - Dic 2016 ISSN 0122-820X E-ISSN 2422-5053 PP: $37-47$ substratos com diferentes texturas superficiais são projetados por programas assistidos por computador (CAD). A fabricação das diferentes superfícies é realizada camada por camada, em um processo por meio da técnica de estereolitografia laser (SLA), diretamente dos arquivos CAD. Resultados: As superfícies dos substratos foram avaliadas por meio de ensaios ópticos, a fim de medir a topografia das superfícies, validar o acabamento superficial e controlar métodos de fabricação por meio das estratégias de medição em diferentes perfis. Conclusão: Este estudo mostrou que as texturas superficiais impressas apresentam uma redução dos valores das medidas de comprimento, volume e massa, em comparação com o definido no desenho.

Palavras-chave: Manufatura aditiva, Microscopia óptica, Resina fotocurável.

\section{Introducción}

La tecnología de manufactura aditiva (AM) se está convirtiendo, cada vez más rápido, en un proceso de fabricación viable debido a las grandes ventajas que aporta en la zona de complejidad del diseño [1]. La AM es ampliamente utilizada en la fabricación de elementos de geometría precisa a partir de datos en tres dimensiones que se obtienen del diseño asistido por computador (CAD) y de la fabricación asistida por computador (CAM), a un ritmo más rápido que la manufactura convencional [2],[3]. En cada proceso de AM, se diseña un modelo en 3D por medio de un software CAD y se exporta a formato STL, que es una malla triangular del objeto, el cual a su vez alimenta las máquinas de impresión 3D con el propósito de construir un sólido a partir de perfiles de capas 2D. Cada nueva capa se une a la capa anterior en la plataforma de construcción hasta que se fabrica una pieza 3D.

Las técnicas principales de la AM son la sinterización selectiva por láser (SLS), estereolitografía (SLA), modelado por deposición fundida (FDM), sinterizado de metal por láser directo (LMD), y las técnicas de impresión 3D (3DP) [4], [5]. Dependiendo del proceso y los materiales utilizados, cada técnica tiene puntos fuertes y débiles. Los parámetros más importantes y apropiados que se deben considerar en la elección de una tecnología AM para un propósito particular son la precisión, tiempo y coste de fabricación. El parámetro de precisión se refiere al espesor de las capas y del sistema de consolidación, teniendo en cuenta que los métodos de fabricación por técnicas de AM no requieren herramientas. El parámetro tiempo de producción se encuentra directamente relacionado con los costes de fabricación por dispositivo [5], [6].

La impresión 3D es una forma de tecnología de fabricación en capas mediante manufactura aditiva, donde un objeto tridimensional es creado a partir de delgadas capas sucesivas que son apiladas formando estructuras 3D [3]. Recientemente, una serie de impresoras tridimensionales (3D) se han desarrollado y son asequibles tanto para usos industriales como personales [7]. Existen principalmente dos tipos de impresoras 3D, la que utiliza repetidamente un proceso de adición de material con bajo punto de fusión, como en el caso del modelado por deposición fundida (FDM) [8], y la estereolitografía (SLA) [9][11] que utiliza resina curable por radiación ultravioleta (UV) [12]-[14]. Algunos de los procesos de estereolitografía han utilizado resina curable por UV con materiales en polvo de cerámica dispersos [15]-[18]. En la actualidad la gama de materiales utilizados en AM es bastante limitado, mientras que hay 
Ely Dannier V-Niño, Jose Luis Endrino-Armenteros, Hugo Armando Estupiñan-Duran, Boris Pérez-Gutiérrez, Andrés Díaz-Lantada

una demanda real en la fabricación de piezas o partes más ligeras con mejores propiedades funcionales [19].

A pesar de la amplia variedad de aplicaciones, la miniaturización y optimización de dispositivos en forma de tres dimensiones, la AM aún no ha alcanzado su pleno potencial, debido principalmente a la falta de técnicas fáciles y rentables de fabricación. Como consecuencia de lo anteriormente expuesto, técnicas estándares de microfabricación, como las técnicas de estereolitografía [20], [21], se han adaptado para la fabricación de productos en 3D usando fotopolímeros. $\mathrm{La}$ Impresión 3D curable por ultravioleta (3DPUV) [22] es una técnica alternativa empleada en la fabricación de microdispositivos basados en fotopolímeros con forma libre de 3D o con características soportadas [23].

De acuerdo a lo anterior, la AM cuenta con un alto potencial para satisfacer las demandas debido al coste del proceso y a la disminución del tiempo en el desarrollo de productos. Por otra parte, las nuevas tendencias industriales como la biónica, construcción de peso ligero o «personalización masiva» son posibles campos de aplicación para la AM [24], [25]. Como consecuencia de la creciente fuerza que ha tenido la AM en el mercado, Roland Berger estima que la AM tendrá una participación de 7,7 millones de euros en el año 2023 [26], donde lo más relevante consistirá en que la calidad de las piezas cumpla con las necesidades de los clientes. En cuanto a la funcionalidad de un componente, la textura de la superficie desempeña un papel importante, ya que los efectos de la superficie causan en general hasta el $10 \%$ de la tasa de defectos en piezas fabricadas [27]. Con respecto a la AM, este valor se estima a ser aún mayor. En consecuencia, una mala calidad de la superficie es una de las principales limitaciones con respecto a esta tecnología [28].
En este trabajo se describen los métodos y materiales utilizados en el diseño y fabricación de determinadas texturas superficiales. Posteriormente se presenta el análisis y las discusiones de los principales resultados obtenidos de la medición y caracterización topográfica de la superficie, los cuales son fundamentales en la industria moderna de fabricación para validar y controlar las estructuras y funcionalidades del área superficial de los dispositivos. El presente estudio permitirá contribuir con las estrategias de medición y proponer algunos desafíos de actualidad en relación con los métodos de fabricación aditiva.

\section{Materiales y métodos}

El proceso de fabricación de los sustratos, con diferentes texturas superficiales, se realizó teniendo en cuenta la identificación y seguimiento de cada una de las etapas presentadas en la Figura 1. El proceso inicia con la definición de los conceptos relacionados con el diseño y las reglas que lo rigen, como por ejemplo la forma geométrica del sustrato y de la superficie y finaliza con la fabricación de los sustratos mediante la técnica de esterolitografía láser. Los softwares de Diseño Asistido por Computadora (CAD) facilitan las actividades de creación, modificación, análisis u optimización de un determinado modelo y la simulación del modelo permite validar las características, propiedades y factibilidad del diseño.
Cúcuta-Colombia 
Jul - Dic 2016

ISSN 0122-820X

E-ISSN 2422-5053

PP: $37-47$

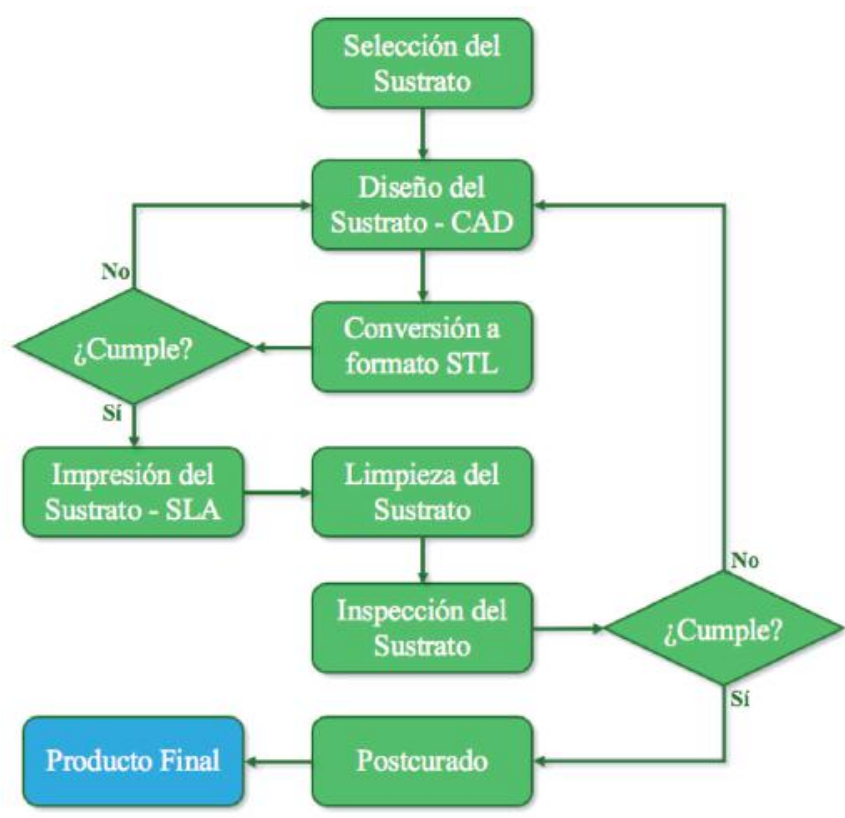

Figura 1. Etapas del proceso en el desarrollo de productos Fuente: Autor

\subsection{Diseño}

Varios programas de CAD y de ingeniería ayudan al proceso de desarrollo de nuevos productos. En nuestro estudio, los sustratos fueron diseñados en SketchUp Make 2016 versión 16.0.19911 (Trimble Navigation Limited). En la Figura 2 se presentan los diseños CADs de los tres (3) tipos de texturas superficiales de geometrías circulares seleccionadas, de sección transversal cuadrada, triangular y de hipérbolas separadas $1 \mathrm{~mm}$ entre secciones.

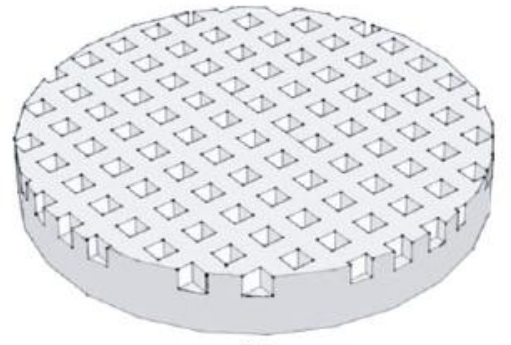

(a)

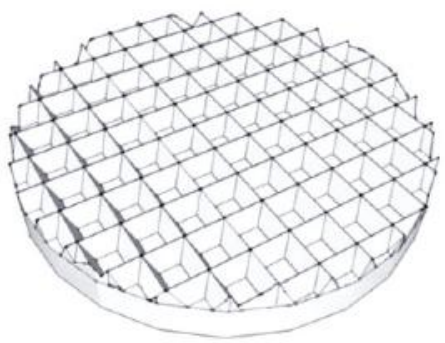

(b)

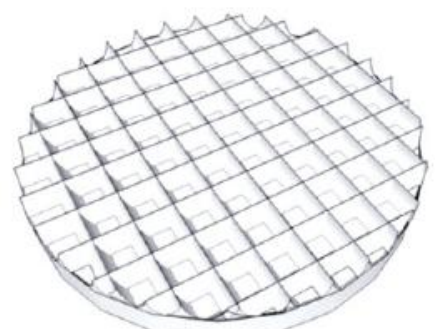

(c)

Figura 2. Sustratos modelados en SketchUp de sección transversal (a) cuadrada, (b) triangular y (c) de hipérbolas Fuente: Autor

Los diseños CADs de las probetas fueron exportados a formato STL (Standard Tessellation Language), formato de archivo requerido por la impresora, y posteriormente fueron validados y verificados usando la aplicación de reparación AutoDesk NetFabb Basic, la cual consiste en corregir los errores geométricos (completar los vacíos encontrados en el sustrato) y así garantizar que las superficies se encuentren cerradas comportándose como un solo sólido.

\subsection{Fabricación}

El material utilizado en el fabricación de los sustratos fue la resina epóxica comercial de nombre Accura ${ }^{\circledR} 60$ (3D Systems, 333 Tres D Systems Circle, rock Hill, SC 29730 
Ely Dannier V-Niño, Jose Luis Endrino-Armenteros, Hugo Armando Estupiñan-Duran, Boris Pérez-Gutiérrez, Andrés Díaz-Lantada

USA). Las propiedades son presentadas en la Tabla I. Es importante resaltar, que en algunas investigaciones enfocadas en evaluar los efectos de los diferentes factores de influencia, tales como, geometría de las piezas, precisión de la máquina, condiciones de procesamiento, tiempo de post-curado o la inclusión de diferentes aditivos y fibras de refuerzo, se han utilizado resinas epóxicas fotocurables, similar a la empleada en nuestro proceso [29]-[32].

La fabricación aditiva, capa por capa, de los sustratos se realizó mediante la técnica de estereolitografía láser en la máquina SLA-3500 de 3D Systems (ver Figura 3), la cual es capaz de leer información sobre geometrías de piezas a partir de los archivos CAD originales exportados en formato STL. La Estereolitografía Láser es un proceso basado en líquido que activa una reacción de polimerización cuando un láser ultravioleta hace contacto con una resina epóxica. El láser UV dibuja, gradualmente, capas en la superficie de la resina líquida, siguiendo una ruta marcada por el archivo STL.

\section{Respuestas}

Cúcuta-Colombia

Vol. 21

No. 2

Jul - Dic 2016

ISSN 0122-820X

E-ISSN 2422-5053

PP: $37-47$

\begin{tabular}{lc}
\multicolumn{2}{c}{ Tabla I. Propiedades de la resina epóxica Accura ${ }^{\circledR} 60$} \\
\hline \multicolumn{1}{c}{ Parámetro } & Valor \\
\hline Densidad & $1.21 \mathrm{~g} / \mathrm{cm}^{3}$ \\
Resistencia a la Tensión & $68-68 \mathrm{MPa}$ \\
Módulo de Young & $2690-3100 \mathrm{MPa}$ \\
Temperatura de Activación $\left(\mathrm{T}_{\text {act }}\right)$ & $58-62^{\circ} \mathrm{C}$ \\
Escala D de Dureza Shore & 86 \\
\hline
\end{tabular}

Fuente: Autor

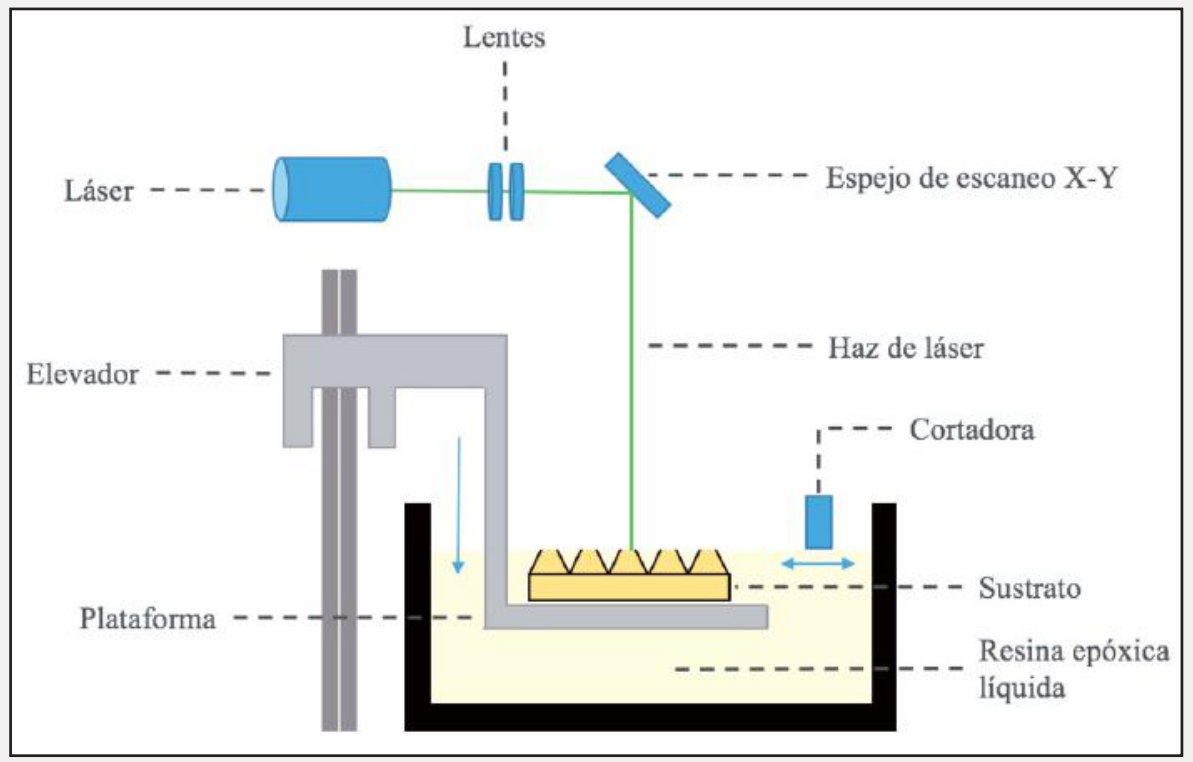

Figura 3. Esquema de la técnica de estereolitografía láser Fuente: Autor

\subsection{Evaluación superficial}

Un proceso de inspección mediante ensayos no destructivos, ayudará a completar este nuevo enfoque, por lo que la evaluación de las texturas superficiales por medio de la inspección visual y microscopía óptica, contribuirá a cuantificar, validar y garantizar 
No. 2

Jul - Dic 2016 ISSN 0122-820X E-ISSN 2422-5053 PP: $37-47$ la calidad de las superficies externas de un objeto fabricado por AM. La microscopía óptica fue realizada con una lente de $10 \mathrm{X}$ en el microscopio Olympus DSX500.

\section{Resultados y análisis}

Los resultados obtenidos de los ensayos no destructivos de inspección visual y microscopía óptica son presentados a continuación.

\subsection{Inspección visual}

En la Figura 4 se muestran los sustratos de diferente textura superficial fabricados en resina epóxica transparente mediante la técnica de estereolitografía láser. Las superficies de textura y contorno en la Figura 4 (a), (b) y (c) se observan bien definidas, sin deformaciones e irregularidades, mientras que en la superficie base se perciben irregularidades debido a la eliminación de los soportes de contacto, entre la plataforma de la impresora y el sustrato, creados durante el proceso de fabricación. Los valores obtenidos de las mediciones de longitud, masa y volumen obtenidos de los sustratos en el diseño CAD y de forma directa después de impresos son presentados en la Tabla II.

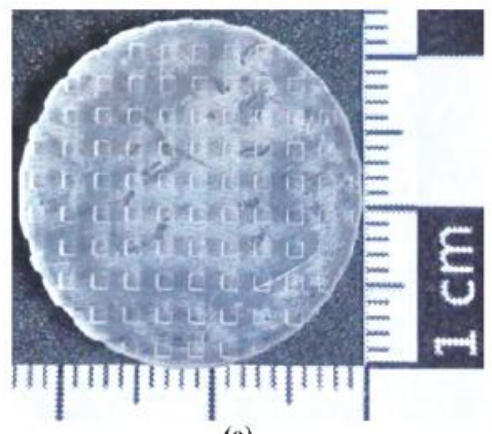

(a)

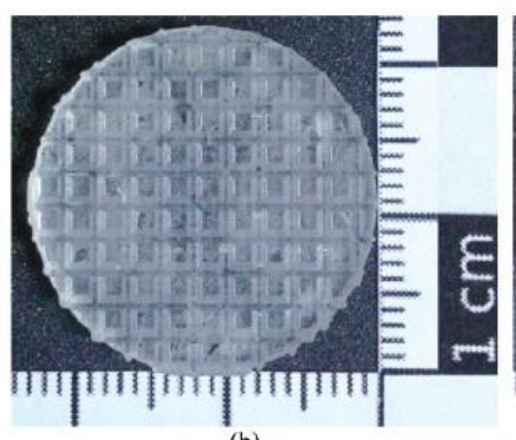

(b)

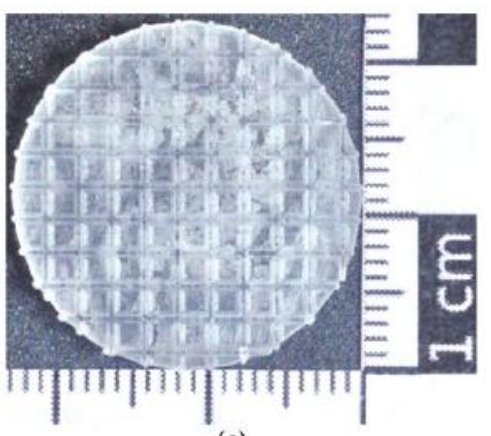

(c)

Figura 4. Sustratos fabricados en resina epóxica de sección transversal (a) cuadrada, (b) triangular y (c) de hipérbolas Fuente: Autor

De los valores reportados en la Tabla II podemos afirmar que las medidas de longitud, masa y volumen de los sustratos fabricados disminuyen en relación con los valores establecidos en el diseño entre el 25 y el $35 \%$. De igual forma apreciamos que el sustrato que presenta un mayor volumen, como es de esperarse, es el sustrato diseñado $\mathrm{y}$ fabricado con una textura superficial con secciones transversales cuadrada seguido del de secciones transversales triangular.

Tabla II. Valores de las medidas de longitud masa y volumen de los sustratos

\begin{tabular}{|c|c|c|c|c|c|c|c|c|}
\hline \multirow{2}{*}{ Sección transversal } & \multicolumn{4}{|c|}{ Mediciones obtenidas del Diseño CAD } & \multicolumn{4}{|c|}{ Mediciones obtenidas del Sustrato Impreso } \\
\hline & $\begin{array}{l}\text { Radio } \\
(\mathrm{cm})\end{array}$ & $\begin{array}{c}\text { Espesor } \\
\mathrm{cm})\end{array}$ & $\begin{array}{c}\text { Volumen } \\
\left(\mathrm{cm}^{3}\right)\end{array}$ & $\begin{array}{l}\text { Masa* } \\
(\mathrm{g})\end{array}$ & $\begin{array}{l}\text { Radio } \\
(\mathrm{cm})\end{array}$ & $\begin{array}{c}\text { Espesor } \\
(\mathrm{cm})\end{array}$ & $\begin{array}{l}\text { Masa } \\
(\mathrm{g})\end{array}$ & $\begin{array}{c}\text { Volumen* } \\
\left(\mathrm{cm}^{3}\right)\end{array}$ \\
\hline Cuadrada & 1.0615 & 0.3 & 0.98 & 1.1858 & 1.0675 & 0.230 & 0.838 & 0.693 \\
\hline Triangular & 1.0630 & 0.3 & 0.86 & 1.0406 & 1.0675 & 0.225 & 0.703 & 0.581 \\
\hline De Hipérbolas & 1.0625 & 0.3 & 0.81 & 0.9801 & 1.0675 & 0.215 & 0.636 & 0.526 \\
\hline
\end{tabular}

\subsection{Microscopía óptica}

En las Figuras 5, 6 y 7, se muestran las imágenes de los sustratos adquiridas en dos regiones de la superficie de textura, en el hueco (ver Figuras 5 (a), 6 (a) y 7 (a)) y en la junta (ver Figuras 5 (b), 6 (b) y 7 (b)). Las medidas registradas en la Figura 5 (a) y (b) manifiestan que las longitudes de las juntas se reducen y los huecos se amplían aproximadamente un $10 \%$ de los valores establecidos en el diseño. 
Ely Dannier V-Niño, Jose Luis Endrino-Armenteros, Hugo Armando Estupiñan-Duran, Boris Pérez-Gutiérrez, Andrés Díaz-Lantada

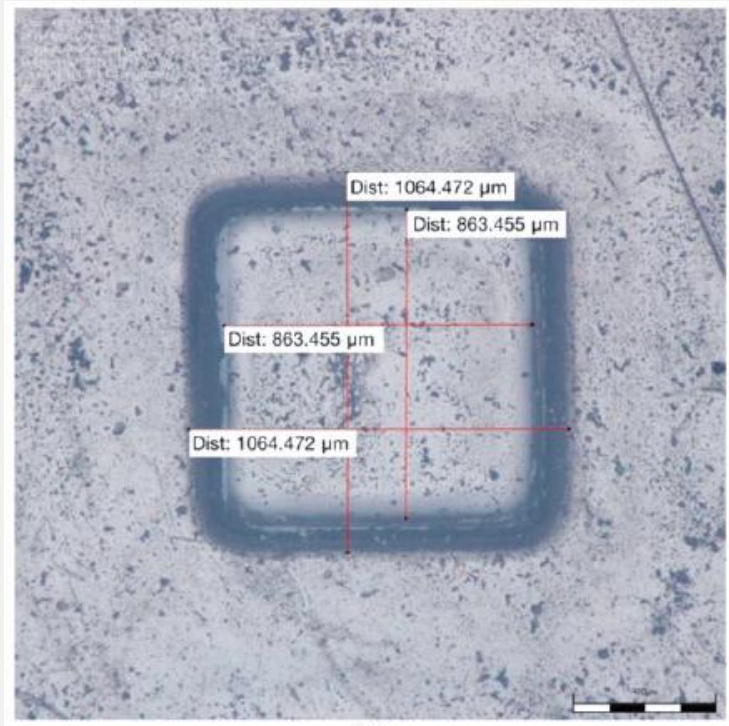

(a)

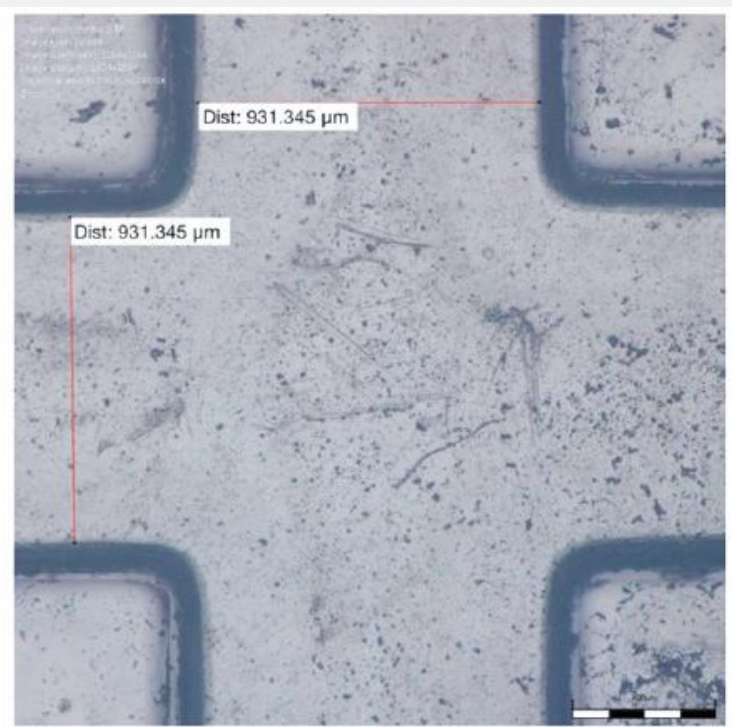

(b)

Figura 5. Imagen adquirida, con lente de 10x, sobre la superficie de textura construida de secciones transversales cuadradas, en el (a) hueco y la (b) junta

Fuente: Autor

En la Figura 6 (a) y (b) correspondiente a la textura superficial formada a partir de secciones transversales de triángulos, se observa que la resolución de la técnica de fabricación por estereolitografía láser sólo logra imprimir hasta aproximadamente un $60 \%$ de la altura de los triángulos generando juntas de aproximadamente 240 micrómetros de longitud, y al igual que lo presentado en la Figura 5, la ampliación de los huecos evidencian que existe una reducción del $10 \%$ de la geometría del sustrato impreso en relación con la definida en el diseño.

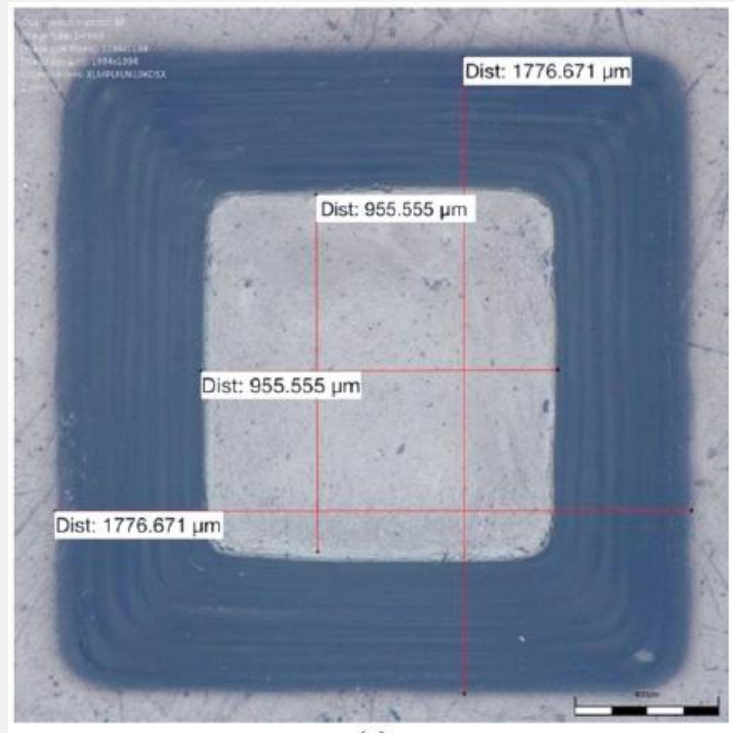

(a)

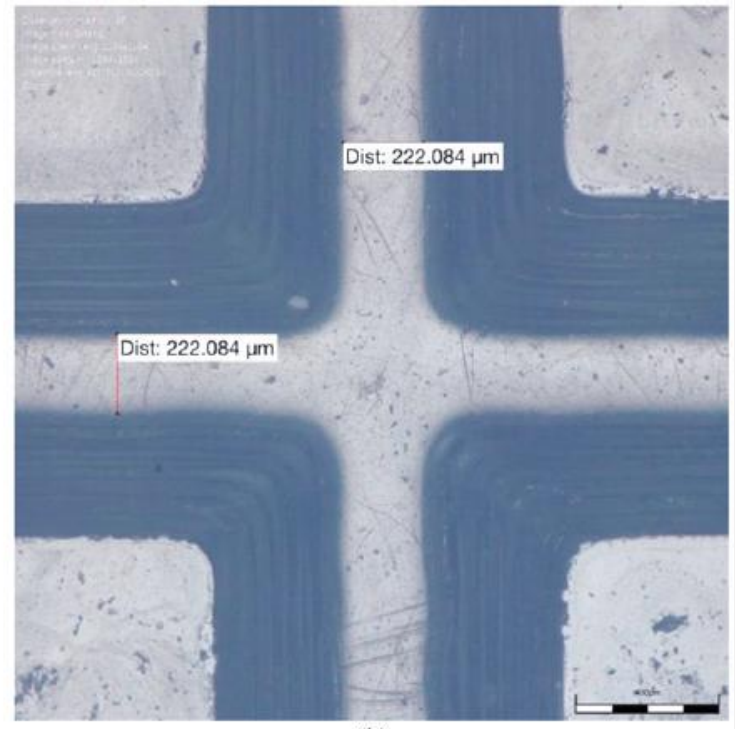

(b)

Figura 6. Imagen adquirida, con lente de 10x, sobre la superficie de textura construida de secciones transversales triangulares, en el (a) hueco y la (b) junta Fuente: Autor 
Jul - Dic 2016 ISSN 0122-820X E-ISSN 2422-5053

PP: $37-47$
El caso de las imágenes adquiridas en las regiones de hueco (ver Figura 7(a)) y junta (ver Figura 7(b)) de la superficie de textura construida con secciones transversales de hipérbolas, se aprecia un mejor detalle en la resolución del proceso de impresión respecto a la textura superficial de secciones transversales de triángulo, el cual puede ser atribuido al decaimiento leve que presenta la superficie, donde la junta tiene una longitud de 180 micrómetros que es menor a la longitud de la junta obtenida de la sección transversal de triángulos (ver Figura 6(b)). Respecto a la altura de las secciones transversales (de hipérbolas, triángulos $\mathrm{y}$ cuadradas) es de esperarse que después de la fabricación presente una disminución del valor establecido en el diseño, debido a los valores de espesores reportados en la Tabla II.

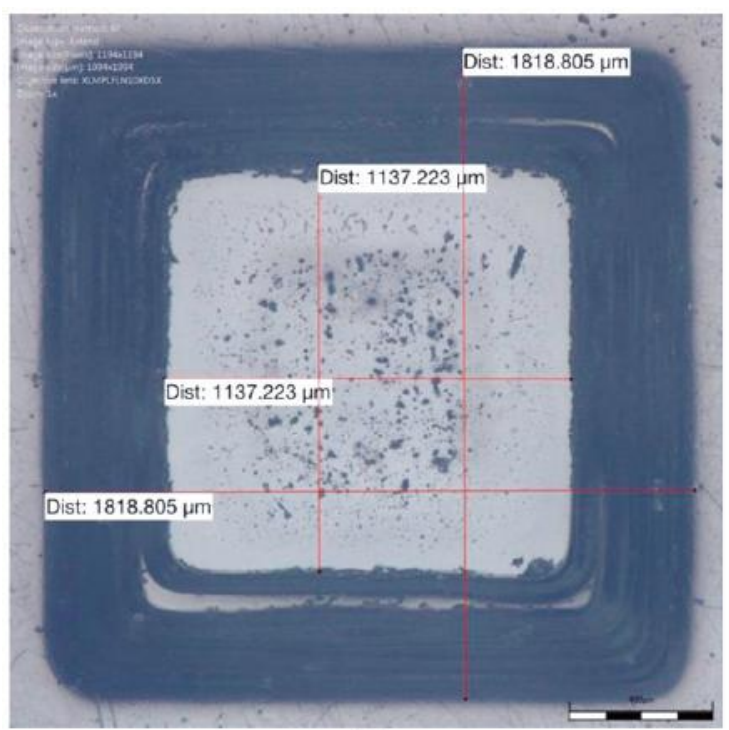

(a)

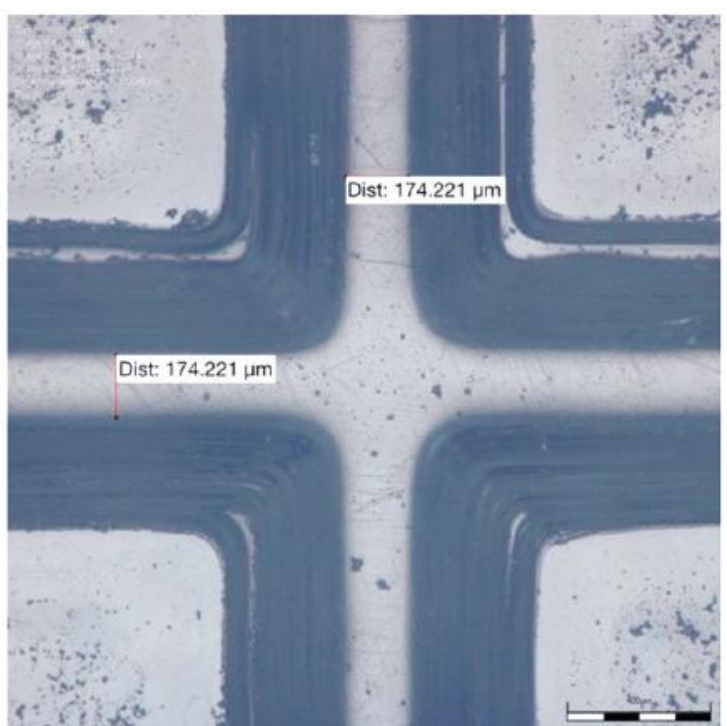

(b)

Figura 7. Imagen adquirida, con lente de 10x, sobre la superficie de textura construida de secciones transversales de hipérbolas, en el (a) hueco y la (b) junta Fuente: Autor

De acuerdo a lo anteriormente presentado, podemos garantizar que las texturas superficiales en los sustratos ayudan a validar el uso de la técnica de estereolitografía láser en el desarrollo de dispositivos micrométricos con geometrías tridimensionales predefinidas alcanzable en los productos finales con la precisión de fabricación.

\section{Conclusiones}

El estudio se ha centrado en presentar un completo proceso de desarrollo de sustratos circulares con diferentes texturas superficiales. Las texturas superficiales se modelaron, por medio del diseño asistido por ordenador y recursos de ingeniería. La fabricación de los sustratos de geometría tridimensionales con los diferentes tipos de textura superficial se realizó, directamente de los archivos CAD exportados en formato STL, por medio de la técnica de estereolitografía láser, proporcionando un excelente compromiso entre el tamaño y la precisión, siendo demostrada mediante microscopía óptica. 
Ely Dannier V-Niño, Jose Luis Endrino-Armenteros, Hugo Armando Estupiñan-Duran, Boris Pérez-Gutiérrez, Andrés Díaz-Lantada

Se encontró que la herramienta Google SketchUp tiene dificultades de diseño en superficies con medidas de longitud del orden de los micrómetros, porque la mínima unidad de longitud disponible son los milímetros. Por tal motivo, en futuros trabajos se han de considerar otros tipos de softwares CADs.

Se observó que la técnica de fabricación por estereolitografía láser imprimió hasta aproximadamente un $60 \%$ de la altura de los triángulos. Luego una pérdida del $40 \%$ es un aspecto a tener en cuenta. Adicionalmente, se evidenció una reducción aproximada del $10 \%$ de la geometría del sustrato impreso en relación con la definida en el diseño, hecho que fue demostrado debido a la ampliación presentada en los huecos de las texturas superficiales.

El enfoque propuesto se puede aplicar en la mejora de las funcionalidades y versatilidad del proceso. Retos en cuanto al uso de micro dispositivos para promover la activación y funcionalización utilizando otros principios físicos y quedar vinculado a la combinación de estrategias tecnológicas para un mejor control del comportamiento de las superficies de los dispositivos, serán temas de estudio para el futuro. Finalmente esperamos que los resultados presentados puedan ser de interés para otros investigadores que llevan a cabo investigaciones en las diversas áreas de la ciencia, ingeniería y tecnología de materiales poliméricos.

\section{Agradecimientos}

La investigación fue realizada con el apoyo financiero de la EU Commission through of the project "Tomax: Tool-less manufacture of complex geometries", under grant agreement number 633192-H2020-FoF-2014-2015/ H2020-FoF-2014.

\section{Referencias}

[1] J. Brennan-Craddock, D. Brackett, R. Wildman and R. Hague. "The design of impact absorbing structures for additive manufacture", Journal of Physics Conference series, vol. 382, pp. 1-7, 2012.

[2] D. W. Hutmacher and M. Sittinger and M. V. Risbud. "Scaffold-based tissue engineering: rationale for computer-aided design and solid freeform fabrication systems", Trends in Biotechnology, vol. 22, pp. 354-362, 2004.

[3] K.G.JayaChristyan, U.Chandrasekhar and K. Venkateswarlu. "A study on the influence of process parameters on the Mechanical Properties of 3D printed ABS composite", Conference Series: Materials Science and Engineering, vol. 114, no. 012109, pp. 1-8, 2016.

[4] D. T. Pham and R. S. Gault. "A Comparison of Rapid Prototyping Technologies", International Journal of Machine Tools and Manufacture, vol. 38, pp. 1257-1287, 1998.

[5] B. Wendel, D. Rietzel, F. Kühnlein, et al. "Additive Processing of Polymers", Macromolecular Materials and Engineering, vol. 293, pp. 799-809, 2008. F. P. Melchels, J. Feijen and D. W. Grijpma. "A review on stereolithography and its applications in biomedical engineering", Biomateriales, vol. 31, pp. 6121-6130, 2010.

[7] K. V. Wong and A. Hernandez. "A Review of Additive Manufacturing", ISRN Mechanical Engineering, vol. 2012, no. 208760, pp. 1-10, 2012.

[8] S. H. Ahn, M. Montero, D. Odell, et al. "Anisotropic material properties of fused deposition modeling ABS" Rapid Prototyping Journal, vol. 8, pp. 248-257, 2002. 
No. 2

Jul - Dic 2016

ISSN 0122-820X

E-ISSN 2422-5053

PP: $37-47$
[9] J. P. Kruth, L. Froyen, J. Van Vaerenbergh, et al. "Selective laser melting of iron-based powder", J. Mater. Process. Technol., Vol. 149, pp. 616-622, 2004.

[10] I. Yadroitsev, P. Bertrand, and I. Smurov. "Parametric analysis of the selective laser melting process", Appl. Surf. Sci., vol. 253, pp. 8064- 8069, 2007.

[11] S. Bremen, W. Meiners, and A. Diatlov. "Laser Technik Journal", Laser Tech. J., vol. 9, pp. 33-38, 2012.

[12] S. Maruo and K. Ikuta. "Submicron stereolithography for the production of freely movable mechanisms by using single-photon polymerization", Sensors and Actuators A: Physical, vol. 100, pp. 70-76, 2002.

[13] C. Sun, N. Fang, D. M. Wu and $\mathrm{X}$. Zhang. "Projection microstereolithography using digital micromirror dynamic mask", Sensors and Actuators A: Physical, vol. 121, pp. 113-120, 2005.

[14] A. T. Pham, D. Kim, T. Lim, et al. "Three-Dimensional SiCN Ceramic Microstructures via NanoStereolithography of Inorganic Polymer Photoresists", Advanced Functional Materials, Vol. 16, pp. 1235-1241, 2006.

46

L. M. Griffith and W. J. Halloran. "Freeform fabrication of ceramics via stereolithography", Journal of the American Ceramic Society, vol. 79, pp. 2601-2608, 1996.

[16] X. Zhang, X. N. Jiang, and C. Sun. "Micro-stereolithographyofpolymeric and ceramic microstructures", Sensors and Actuators A physical, vol. 77, 149-156, 1999.

[17] C. Hinczewski, S. Corbel, and T. J. Chartier. "Ceramic suspensions suitable for stereolithography", Journal of the European Ceramic Society, vol. 18, pp. 583-590, 1998.

[18] F. Tsumori, H. Kawanishi, K. Kudo, et al. "Development of threedimensional printing system for magnetic elastomer with control of magnetic anisotropy in the structure", Japanese Journal of Applied Physics, vol. 55, no. 06GP18, pp. 1-5, 2016.

[19] S. Kenzari, D. Bonina, J. M. Dubois and V. Fourné. "Complex metallic alloys as new materials for additive manufacturing", Science and Technology of Advanced Materials, vol. 15, no. 024802, pp. 1-9, 2014.

[20] R. Liska, M. Schuster, R. Inführ, et al. "Photopolymers for rapid prototyping", Journal of Coatings Technology and Research, vol. 4, pp. 505-510, 2007.

[21] S. Kawata , H. B. Sun, T. Tanaka, and K. Takada. "Finer features for functional microdevices", Nature, vol. 412, no. 6848, pp. 697-698, 2001.

[22] L. L. Lebel, B. Aissa, M. A. El Khakani andD. Therriault. "Ultraviolet-assisted direct-write fabrication of carbon nanotube/polymer nanocomposite microcoils", Advanced Materials, vol. 22, pp. 592-596, 2010.

R. D. Farahani, L. L. Lebel and D. Therriault "Processing parameters investigation for the fabrication ofselfsupported and freeform polymeric microstructures using ultravioletassisted three-dimensional printing", Journal of Micromechanics and Microengineering, vol. 24, pp. 1-12, 2014. 
Ely Dannier V-Niño, Jose Luis Endrino-Armenteros, Hugo Armando Estupiñan-Duran, Boris Pérez-Gutiérrez, Andrés Díaz-Lantada

[24] J. Breuninger, R. Becker, A. Wolf, S. Rommel and A. Verl. Generative Fertigung mit Kunststoffen. Berlin: Springer, 2013.

[25] T. Grimm, G. Wiora and G. Witt. "Characterization of typical surface effects in additive manufacturing with confocal microscopy", Surface Topography: Metrology and Properties, vol. 3, pp. 1-12, 2015.

[26] R. Berge. Strategy Consultants GmbH. Frankfurt. "Additive Manufacturing. A Game Changer for the Manufacturing Industry?", 2013, [Online]. Disponible en: www.rolandberger.com/media/ pdf/Roland_Berger_Additive_ Manufacturing_20131129.pdf

[27] R. Leach. Optical Measurement of Surface Topography, Berlin: Springer, 2011.

[28] S. Thomas, S. Ernst and S. Michael. "Material optimization of PA12 laser sintering powder to improve surface quality", in ANTEC Conference proceedings (Charlotte, North Carolina, US), vol. 4, pp. 1910-1914, Society of Plastics Engineers, 2006.

[29] K. Chockalingam, N. Jawahar and U. Chandrasekhar. "Influence of layer thickness on mechanical properties in stereolithography", Rapid Prototyping Journal, vol. 12, no. 2, pp. 106-113, 2006.

[30] B. Widemann, K. H. Dusel and J. Eschl. "Investigation into the influence of material and process on part distortion", Rapid Prototyping Journal, vol. 1, pp. 17-22, 1995.

[31] G. V. Salmoria, C. H. Ahrens, M. Fredel, V. Soldi and A. T. Pires. "Stereolithography somos 7110 resin: Mechanical behavior and fractography of parts post-cured by different methods", Polymer Testing, vol. 24, pp. 175-162, 2005.

[32] J. H. Sandoval and R. B. Wicker. "Functionalizing stereolithography resins: effects of dispersed multiproperties" Rapid Prototyping Journal, vol. 12, pp. 292-303, 2006. walled carbon nanotubes on physical

properties" Rapid Prototyping
Journal, vol. 12, pp. 292-303, 2006.

